\title{
A Review of Anesthetic Effects on Renal Function: Potential Organ Protection
}

\author{
Negar Motayagheni ${ }^{\mathrm{a}} \quad$ Sheshanna Phan $^{\mathrm{b}}$ Crystal Eshraghi $^{\mathrm{b}} \quad$ Ala Nozari $^{\mathrm{c}}$ \\ Anthony Atala ${ }^{\mathrm{d}}$ \\ anstitute for Regenerative Medicine (Wake Forest Institute of Regenerative Medicine), Wake Forest School \\ of Medicine Medical Center Boulevard, Winston-Salem, NC, USA; b Department of Anesthesiology, Division of \\ Molecular Medicine, UCLA David Geffen School of Medicine, Los Angeles, CA, USA; ' Department of Anesthesia, \\ Critical Care and Pain Medicine, Massachusetts General Hospital, Harvard Medical School, Boston, MA, USA; \\ ${ }^{d}$ Institute of Regenerative Medicine, Wake Forest School of Medicine, Winston-Salem, NC, USA
}

\section{Keywords}

Anesthetic drugs · Ischemia-reperfusion injury · Volatile anesthetics - Organ protection - Transplant · Intravenous anesthetics - Lipid emulsion - Propofol - Cardioprotection . Renal protection

\begin{abstract}
Background: Renal protection is a critical concept for anesthesiologists, nephrologists, and urologists, since anesthesia and renal function are highly interconnected and can potentially interfere with one another. Therefore, a comprehensive understanding of anesthetic drugs and their effects on renal function remains fundamental to the success of renal surgeries, especially transplant procedures. Some experimental studies have shown that some anesthetics provide protection against renal ischemia/reperfusion (IR) injury, but there is limited clinical evidence. Summary: The effects of anesthetic drugs on renal failure are particularly important in the context of kidney transplantation, since the conditions of preservation following removal profoundly influence the recovery of organ function. Currently, preservation procedures are typically based on the usage of a cold-storage solu-
\end{abstract}

tion. Some anesthetic drugs induce anti-inflammatory, antinecrotic, and anti-apoptotic effects. A more thorough understanding of anesthetic effects on renal function can present a novel approach for developing organ-protective strategies. The aim of this review is to discuss the effects of different anesthetic drugs on renal function, with particular focus on IR injury. Many studies have demonstrated the organ-protective effects of some anesthetic drugs, specifically propofol, which indicate the potential of some anesthetics to introduce novel organ protective targets. This is not surprising, since lipid emulsions are major components of propofol, which accumulating data show provide organ protective effects against IR injury. Key Messages: Thorough understanding of the interaction between anesthetic drugs and renal function remains fundamental to the delivery of safe perioperative care and to optimizing outcomes after renal surgeries, particularly transplant procedures. Anesthetics can be repurposed for organ protection with more information about their effects, especially during transplant procedures. Here, we review the effects of different anesthetic drugs - specifically those that contain lipids in their structure, with special reference to IR injury.

(c) 2017 S. Karger AG, Base

\section{KARGER}

(c) 2017 S. Karger AG, Basel 


\section{Introduction}

Renal ischemia/reperfusion (IR) injury is a leading cause of preoperative acute kidney injury (AKI), which frequently complicates major vascular, cardiac, transplant, and liver surgeries [1]. AKI has been shown to occur after some major surgeries, raising questions regarding the role of the operative procedures - including the administration of anesthesia and its effects on renal function [1].

There is contradictory evidence regarding the effects of anesthetics on renal function. Some studies have shown that the administration of some types of anesthesia during surgery, as well as surgical stress itself, can affect renal function. Indirect effects are more pronounced than the direct effects [2]. However, other studies have shown that some anesthetic drugs induce anti-inflammatory, anti-necrotic, and anti-apoptotic effects that protect against AKI [3]. This raises several questions, including: Can anesthetics attenuate or prevent renal IR injury? What are the possible mechanisms? How does the anesthetic technique influence patient outcomes after renal transplantation? Can we repurpose them as organ-protective agents?

Identifying appropriate anesthetic technique for renal procedures, especially transplantations, is vital (Table 1). In particular, novel interventions that protect against IR injury are needed to improve early graft function after kidney transplantation. Available general and local anesthetics, including third generation inhaled anesthetics, propofol, and amide-class local anesthetics, are effective and safe with a low incidence of side effects. Anesthetics seem to exhibit an organ-protective potential via multiple different mechanisms, including reducing IR injury (Fig. 1) [4]. Some researchers propose that these anesthetics prevent the uncontrolled opening of the mitochondrial permeability transition pore after ischemia, which leads to the release of pro-apoptotic factors and necrotic cell death [5]. In particular, propofol, a widely used anesthetic, has shown potential as a novel organ-protective agent through its efficient membrane-targeted and cytoprotective effects [4]. This is not surprising since lipid emulsions are major components of propofol, which accumulating data show provide organ protective effects against IR injury in many organs, such as the heart, kidney, liver, and intestines [6-13].

In this review, we summarize the research history on the effects of anesthetic drugs on kidney function, including cellular mechanisms of anesthetic-mediated protection in different organs (Table 2).

Anesthetic Effects on Renal Function

\section{General Anesthesia}

\section{Volatile Anesthetics}

Volatile anesthetics are administered to many patients subjected to general anesthesia and are an integral part of the perioperative period. Methoxyflurane was the first nonflammable halogenated volatile anesthetic gas synthesized [3]. Methoxyflurane caused dose-dependent abnormalities post-surgery, including vasopressin-resistant polyuria, serum hyperosmolality, hypernatremia, increased concentrations of serum urea nitrogen and inorganic fluoride, and decreased urinary potassium, sodium, osmolality, and urea nitrogen concentrations, with clinical toxicity at dosages greater than $90 \mu \mathrm{mol} / \mathrm{L}$ [14]. Consequently, nephrotoxicity induced by methoxyflurane was generalized to all halogenated anesthetics. However, most third-generation inhaled anesthetics are effective and safe [4].

Fluorinated anesthetics, specifically sevoflurane and enflurane, did not cause deterioration of postoperative renal function in patients with preexisting renal issues [15]; none of the patients needed dialysis or had permanent deterioration of renal insufficiency. Furthermore, both animal and human studies have demonstrated that neither the duration of systemic fluoride exposure nor the fluoride peak values corresponded to anesthetic nephrotoxicity [3]. In fact, the metabolism of enflurane to inorganic fluoride during and after surgery did not cause a clinically significant level of renal disease or dysfunction [16].

Accumulating data show protective effects of some volatile anesthetic drugs against IR injury $[17,18]$. In a study analyzing data from the past 15 years, pre-injury administration of a volatile anesthetic was shown to decrease the impact of IR injury on the heart, brain, and kidney [19]. Other data demonstrated that volatile agents administered shortly after injury can decrease IR injury [20].

Additionally, Darwin's theory of evolution led to the concept of preconditioning, a mechanism in which brief sublethal periods of ischemia provide protection from a subsequent lethal episode of ischemia and mitigate the effect of IR [21]. Subsequently, the organism or the tissue will acquire an "injury-resistant" phenotype for a certain period [22]. Interestingly, brief periods of ischemia at the onset of reperfusion are associated with cardioprotection, leading to a decrease in myocardial infarction size [23]. Similarly, isoflurane has been shown to improve remodeling after coronary artery occlusion in rats [24]. Preconditioning is now a well-established property of volatile 
Table 1. Overview of common anesthetic agents with their primary physiological and adverse effects

\begin{tabular}{|c|c|c|}
\hline Drug & Action & Adverse effects \\
\hline $\begin{array}{l}\text { Volatile anesthetics } \\
\text { Methoxyflurane }\end{array}$ & Analgesic & $\begin{array}{l}\text { Respiratory and cardiovascular system depression, renal damage no longer } \\
\text { available for use in the United States }\end{array}$ \\
\hline Sevoflurane & Anesthetic & Raises intracranial pressure \\
\hline Enflurane & Anesthetic & Increased risk of seizure activity, malignant hyperthermia \\
\hline $\begin{array}{l}\text { Intravenous anesthetics } \\
\text { Ketamine }\end{array}$ & Anesthetic Analgesic & Psychomimetic effects post-surgery \\
\hline Dexmedetomidine & Anesthetic Analgesic & $\begin{array}{l}\text { Use with caution in patients with preexisting cardiac conduction defects, } \\
\text { bradycardia, hypovolemia }\end{array}$ \\
\hline Lidocaine & Analgesic & \\
\hline
\end{tabular}

anesthetics, specifically of sevoflurane; these anesthetics are recommended as the agents of choice by the American Heart Association for high-risk patients. Preconditioning and postconditioning with sevoflurane exert a significant protective effect against IR injury in the rat lung transplantation model [25].

Other volatile anesthetics exhibit promising post-conditioning properties after cardiac surgery. At the basic level, the myocardial protective effects of sevoflurane involve apoptotic mRNA inhibition, neuromodulation, cytokine/inflammation modulation, redox-sensitive pathways, endothelial preservation, ion channels, and notch signaling pathways [26]. These findings open a new field of investigation for potential therapies aimed to diminish secondary organ injury, as well as transplants. However, more studies are required to assess the magnitude of collateral protection of other organs.

Additionally, researchers have looked at the possibility of adding volatile anesthetics to preservation solutions for renal transplantations. Such anesthetic management aims to maintain the optimum perfusion pressure of the renal allograft in order to preserve its function. Both sevoflurane and enflurane have been shown to undergo biodegradation into inorganic fluoride. Evidence of renal concentrating ability and renal tubular injury with transient impairment was found in patients who received sevoflurane and enflurane [27], as a serum fluoride concentration of $50 \mu \mathrm{mol} / \mathrm{L}$ is the peak nephrotoxicity value.
Furthermore, sevoflurane can impair kidney function; the inorganic fluoride ions resulting from its defluorination and the production of compound A from the reaction with carbon dioxide absorbent have been associated with nephrotoxicity $[28,29]$. Fluoride levels following the administration of isoflurane or halothane increase by $3-5$ and 1-2 $\mu \mathrm{mol} / \mathrm{L}$, respectively, causing the risk of nephrotoxicity to be relatively improbable. Comparatively, desflurane's resistance to biodegradation allows even prolonged exposure to be associated with normal renal function [28]. In a recent study, researchers stored porcine kidneys in the preservation solution Celsior, which was saturated with argon or xenon. Argon-Celsior showed promise in renal transplant preservation by improving early functional recovery, graft quality, and survival, in comparison to Xenon-Celsior [30].

Recent studies have shown that volatile anesthetics provide protective effects during and after ischemic and inflammatory conditions that occur in the perioperative period by modulating IR injury and inflammation $[3,15$, $16,31]$. Researchers found that isoflurane provides preconditioning renoprotective effects through anti-inflammatory and anti-apoptotic actions in a rat model [32]. Isoflurane may be protecting against renal tubular necrosis and inflammation by inducing renal tubular CD73 and adenosine generation, which is dependent on transforming growth factor-beta 1 [33]. Similarly, sevoflurane pretreatment enhanced hypoxia-inducible factor- $2 \alpha$ ex- 
Fig. 1. Hypothesized mechanisms for protective effects of anesthetics.

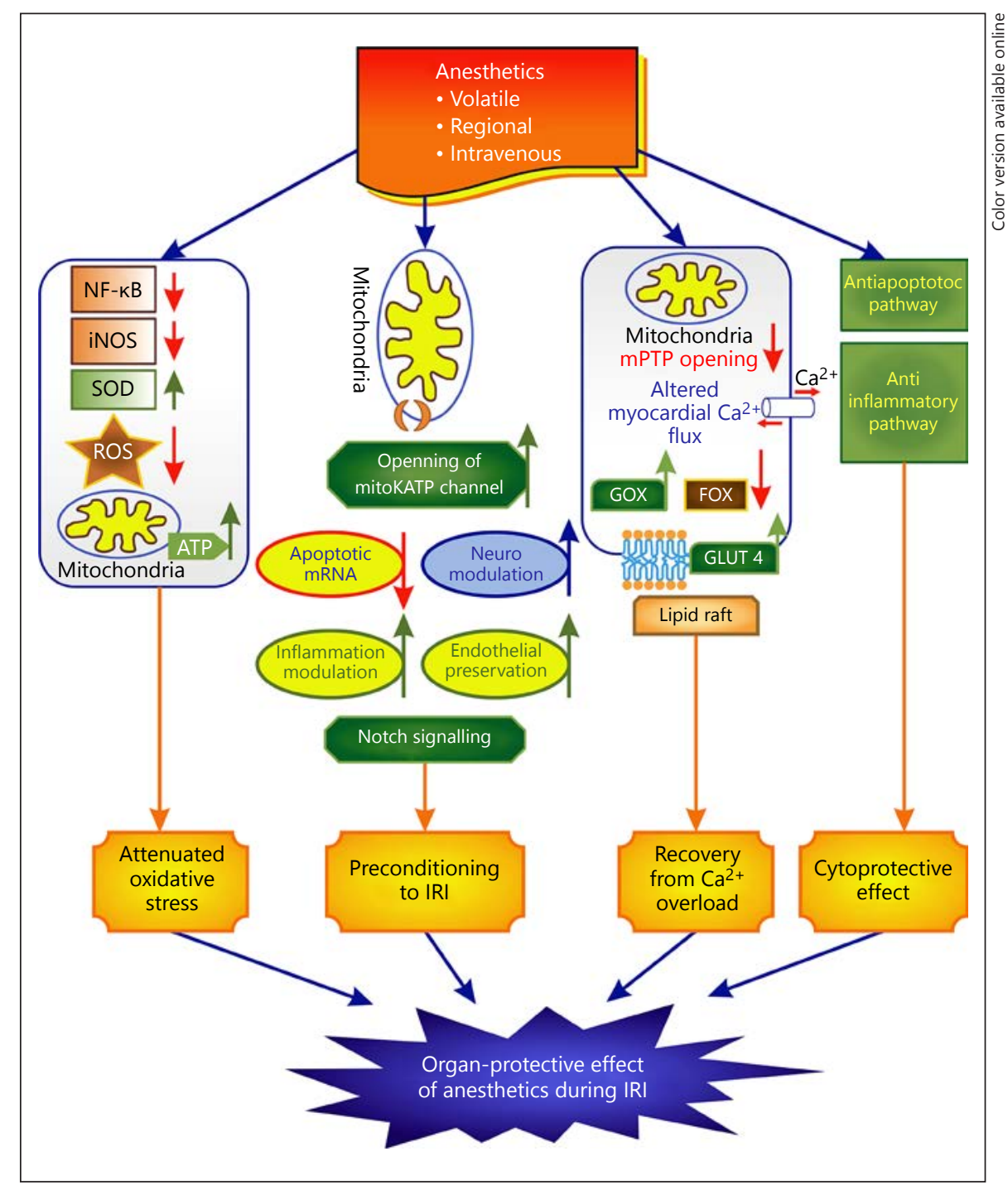

pression in a mouse model of renal IR injury [34]. Another study showed that xenon protects against AKI by activating miR-21 target signaling pathways [35].

Additionally, a recent study simulating liver transplantation in rats investigated the mechanisms by which volatile anesthetics yield their organ protective effects, comparing the protective and antioxidant properties of sevoflurane and isoflurane [36]. It was found that sevoflurane had significantly better protective and antioxidant effects during both cold preservation and the early phases of organ reperfusion in comparison to isoflurane, suggesting the differential protective effects of common volatile anesthetics [36].

There is significant clinical evidence for volatile anesthetic-mediated organ protection. A recent study showed that sevoflurane preconditioning significantly lessened the postoperative rise of transaminase levels in patients undergoing liver resection [37]. Furthermore, a short period of sevoflurane preconditioning in patients undergoing coronary artery bypass graft surgery was shown to significantly decrease both the release of a myocardial contractile dysfunction marker and the levels of plasma cystatin $\mathrm{C}$ concentrations [38]. These findings suggest that sevoflurane causes improvements in renal and cardiac function following major surgery. Additionally, a recent study suggested that during the early postoperative period following kidney transplantation, the estimated glomerular filtration rate improves significantly when living donors are anesthetized with a volatile anesthetic, as compared to with propofol [39]. The protective effects 
Table 2. Proposed interactions and mechanisms of anesthetics

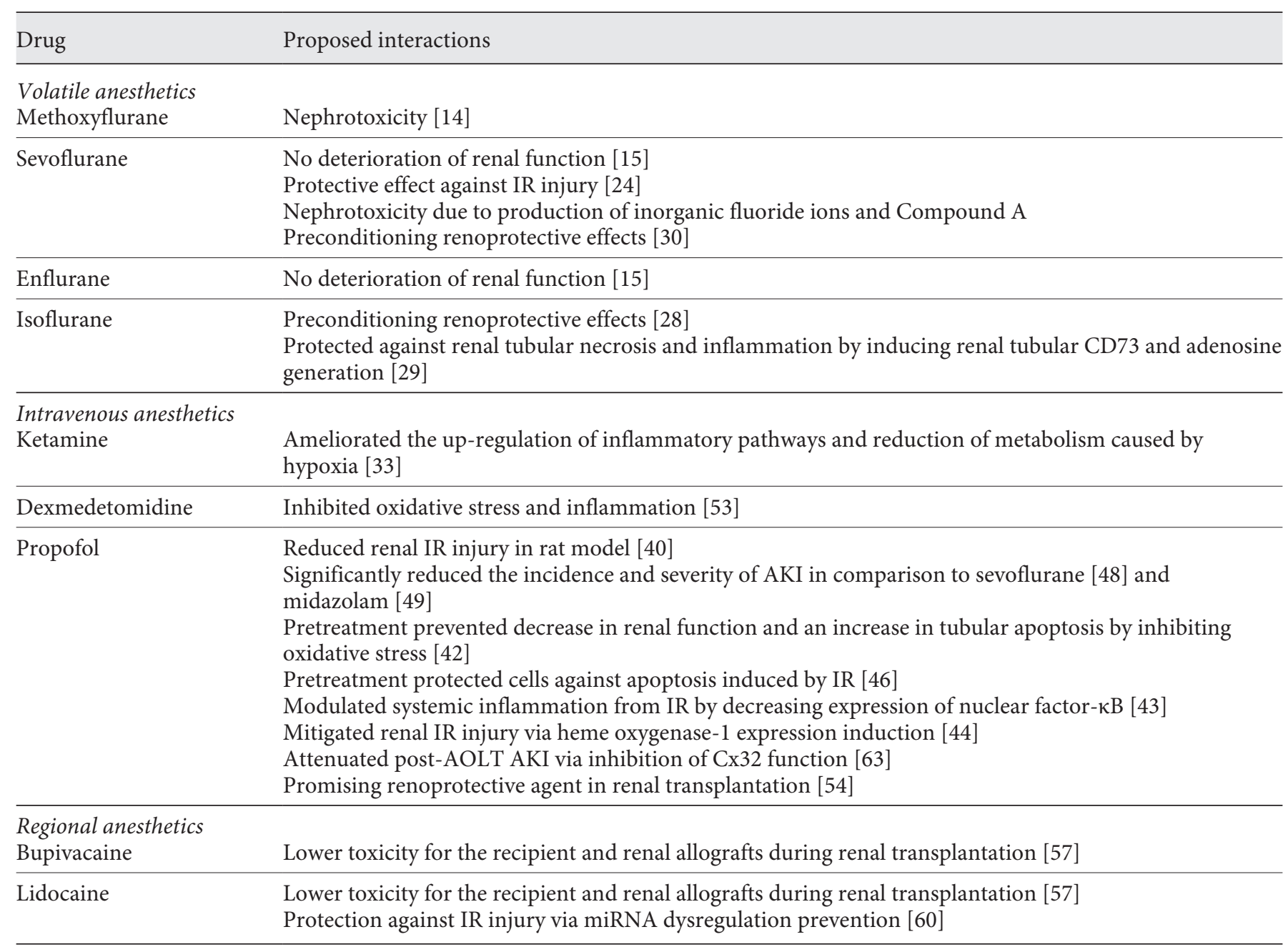

of volatile anesthetics were also evaluated in a recent study that investigated the mechanisms of IR injury, and identified one such mechanism as intracellular calcium overload. It was suggested that such anesthetics may protect the myocardium from IR injury by altering myocardial calcium fluxes, preserving myocardial energetics, and protecting the region from reactive oxygen species injury [40]. The relatively higher efficiency of enflurane and halothane in comparison to isoflurane regarding these effects has been explained by their effect on myocardial cells: halothane and enflurane primarily decrease intracellular calcium levels by directly acting on the sarcoplasmic reticulum, while isoflurane only decreases transsarcolemnal calcium entry (Fig. 1) [40].

Recently, various studies have shown the protective effects of volatile anesthetics in terms of renal IR injury.
One study determined that volatile anesthetics, such as isoflurane, provide IR injury protection by attenuating both inflammation and necrosis [41]. Another study showed that preconditioning with xenon had a protective effect by preventing renal IR injury from progressing to AKI due to its natural induction of hypoxia-inducible factor, thus yielding potentially important clinical applications [42]. Furthermore, another study investigating histological tubular cell damage in a rabbit model found that desflurane preconditioning reduced renal IR injury via its protective effect on the kidneys [43].

Although some volatile anesthetics attenuate AKI, multiple studies have shown that propofol, an intravenous anesthetic with anti-inflammatory properties, may attenuate AKI more effectively. 


\section{Intravenous Anesthetics}

Renal IR injury is a risk factor for acute renal failure and delayed graft function. Pathogenic factors for renal IR include, but are not limited to, the following: oxidative stress, inflammation, cellular necrosis, and apoptosis [44]. Some anesthetic drugs induce anti-inflammatory, anti-necrotic, and anti-apoptotic effects through different mechanisms [3]. In a recent study, researchers intravenously administered ketamine into fetal sheep before inducing hypoxia. Ketamine ameliorated the upregulation of inflammatory pathways and reduction of metabolism caused by hypoxia [45]. Furthermore, thiopental pretreatment reduced renal IR injury induced by free radicals $[46,47]$. Reinforcing this finding, dexmedetomidine has been shown to decrease brain IR injury and inhibit nuclear factor- $\mathrm{\kappa B}$ and intercellular adhesion molecule 1 expression through the inhibition of oxidative stress and inflammation, which are pathogenic factors of IR injury [48]

However, the majority of research studies have focused on the non-anesthetic effects of propofol, such as its antioxidant, immunomodulatory, analgesic, and neuroprotective effects [47]. Propofol has been shown to have protective effects against IR injury in multiple organs, including the heart, kidneys, liver, and intestines [6-13, 49-52].

The antioxidant abilities of propofol significantly reduced IR injury in a rat model of renal IR [53]. Researchers have shown that pretreatment with propofol attenuated the characteristic decrease in renal function and an increase in tubular apoptosis in male rat kidneys subjected to IR. Propofol protected cells from apoptosis by inhibiting oxidative stress and the corresponding downstream pathways, such as mitochondrial stress [54]. Propofol may counteract oxidative stress by lowering the formation of $\mathrm{F}(2)$-isoprostane, a marker of oxidative stress, during transplantation and post-surgery [55]. Propofol also modulates systemic inflammation resulting from IR by decreasing the expression of nuclear factor$\kappa \mathrm{B}$, a nuclear transcription factor that plays a key role in oxidative stress (Fig. 1) [56]. Propofol may also induce heme oxygenase- 1 expression in order to mitigate renal IR injury [57]. Furthermore, propofol can also induce protection against renal IR injury aggravated by hyperglycemia through its antioxidant abilities [58]. In comparison to etomidate, propofol significantly attenuated tubular damage after reperfusion in hyperglycemic rats. It also preserved superoxide dismutase levels and attenuated post-reperfusion increases in levels of myeloperoxidase, interleukin- $1 \beta$, tumor necrosis factor- $\alpha$, production of inducible nitric oxide synthase, and phosphorylation of inhibitor of $\kappa \mathrm{B}$ and nuclear factor- $\kappa \mathrm{B}$ [58].

Propofol may protect cells against apoptosis induced by IR through a preconditioning effect. In one study, propofol attenuated IR injury in LLC-PK1 cells when presented either 1 or $24 \mathrm{~h}$ before IR or during the recovery period but not when it was added only during ischemia. This effect may have been partially mediated by $\mathrm{K}_{\mathrm{ATP}}$ channels [59]. In addition, propofol pretreatment may modulate bone morphogenetic protein 2 expression to reduce renal oxidative injury and facilitate repair following IR injury [60].

In a comparison between patients receiving propofol or sevoflurane during cardiopulmonary bypass, propofol significantly reduced the incidence and severity of AKI (which was defined using the AKI network criteria as an absolute increase in serum creatinine of $0.3 \mathrm{mg} / \mathrm{dL}$, an increase $\geq 150 \%$ from the baseline value, or as a urine output of $<0.5 \mathrm{~mL} / \mathrm{kg} / \mathrm{h}$ for $>6 \mathrm{~h}$ within $48 \mathrm{~h}$ postoperative) in comparison to sevoflurane [61]. This suggests that propofol may be a better renoprotective agent for cardiopulmonary bypass than sevoflurane. No relevant clinical complications occurred with either anesthetic method, and other postoperative outcomes (such as intensive care unit [ICU] stay and in-hospital mortality) were similar between the 2 groups. AKI that occurred in patients who received propofol was confined to the lowest stage of the AKI Network criteria, while AKI that occurred in patients who received sevoflurane varied across the disease spectrum. In fact, significantly more patients receiving sevoflurane displayed severe renal dysfunction and required dialysis. Measured perioperative changes in serum biomarkers of renal injury and inflammatory mediators, and the occurrence of postoperative complications contributed to the clinical evaluation of patient outcomes. C-reactive protein, creatine kinase-MB, and neutrophil counts were measured pre- and postoperatively, and interleukin-1, interleukin-6, and tumor necrosis factor- $\alpha$ levels were assessed to evaluate the degree of inflammation and AKI [61]. In another study, patients treated with propofol had better ICU survival than those treated with benzodiazepine. The outcomes for patients in the ICU that were exclusively treated with propofol or midazolam were compared for the first $48 \mathrm{~h}$. Patients treated with propofol had a lower risk of AKI, fluid-related complications, and less need for RRT compared with midazolam [62].

It has been suggested that reperfusion-induced enhancement of the connexin32 (Cx32) gap junction plays an important role in mediating AKI post-liver transplan- 
tation. In one study, male rats underwent autologous liver transplantation (AOLT) with or without selective Cx32 inhibitor, 20-amino-ethoxydiphenyl, or propofol. AOLT significantly increased renal Cx32 protein expression, gap junction formation, and oxidative stress, and it impaired renal function. Propofol inhibited Cx32 function while attenuating post-AOLT AKI [63]. Another study suggested that propofol exerts a renoprotective effect against AKI after orthotopic liver autotransplantation through the upregulation of the nuclear factor, erythroid 2-related factor 2, a regulator of the cellular-defense response protection against oxidative injury [64]. A recent study found that propofol protects against hepatic IR injury through the regulation of mitogen-activated protein kinase 6 expression by miR-133a-5p [65]. There is extensive supporting data regarding the protective effects of lipid emulsions against IR injury [6-13]. Considering the presence of lipids in propofol, this could provide new insights into organ-protective targets.

Additionally, researchers have evaluated the effectiveness of propofol in preservation solution in a porcine autotransplantation model after $45 \mathrm{~min}$ of ischemia. Their results demonstrated that propofol is a promising renoprotective agent that may attenuate hypothermic and ischemic AKI in renal transplantation through its antioxidant effects [66]. This mechanism of transplantation preservation is driven by propofol's action as a cytoprotective agent and membrane-targeted antioxidant. As such, propofol protects tubular cells from being affected by hypothermic injury in vitro, and the addition of its cyclodextrin complex to the preservation fluid during machine perfusion delivers it to the kidneys and prevents lipid peroxidation, while diminishing the early reperfusion period's renovascular resistance after transplantation [66]. However, recent clinical trials have suggested that volatile anesthetics, such as desflurane, used for anesthetic maintenance and preservation during transplantation are associated with better outcomes than intravenous anesthetics [67]. In fact, most currently available volatile anesthetics have been shown to efficiently preserve hepatic function and blood flow [68].

Furthermore, a recent study involving rats with obstructive jaundice evaluated the protective effects of various common intravenous anesthetics on renal tissues, and found that the incidence of postoperative AKI was higher in rats with obstructive jaundice than in those without it, and that obstructive jaundice causes renal tissue to become sensitive to anesthetic damage [69]. It was found that propofol and ketamine were the 2 anesthetic agents that produced the least renal oxidative stress in this model of common bile duct ligation-induced obstructive jaundice [69]. Free radical injury in renal tissue during transplantation surgery was suggested to have significant importance in preventing related AKI.

Various clinical studies have helped elucidate the relationship between anesthesia and clinical AKI. A recent meta-analysis was conducted for remote ischemic preconditioning (RIPC) trials in patients prior to undergoing cardiac surgery. Although researchers found that there was a significant reduction in AKI when propofol was not used, no effects were observed in those who received both propofol and RIPC during surgery. This suggests that propofol may interact with the protective components of RIPC, thereby improving clinical outcomes [70]. Furthermore, another recent study compared the clinical outcomes of propofol and sevoflurane anesthesia on postoperative AKI [71]. A multivariable analysis of 4,320 colorectal surgery patients' medical records revealed that the incidence of AKI was significantly higher in patients who received sevoflurane than in those who received propofol, suggesting that anesthesia with propofol may be associated with improved clinical AKI outcomes for patients [71]. Additionally, another study consisting of a randomized placebo-controlled trial investigated whether dexmedetomidine prevents AKI following valvular heart surgery [72]. AKI incidence, morbidity, and ICU stay were all found to be significantly lower for patients who received dexmedetomidine than for those who received the placebo, suggesting that perioperative administration of dexmedetomidine may reduce the incidence and severity of AKI, thus improving clinical outcomes for patients undergoing cardiac surgery [72].

\section{Regional Anesthesia}

It has been found that the addition of bupivacaine to a depolarizing cardioplegia solution reduced cell damage and improved cardiac function after prolonged storage [73]. Bupivacaine's ability to decreased cell damage suggests that it may have some protective effects against renal IR injury.

In fact, several studies have found that epidural analgesia (EDA) reduces the incidence of acute renal failure. In a study that investigated the effect of EDA on renal blood flow in 13 healthy volunteers, researchers administered lidocaine $2 \%$ without epinephrine to establish a bilateral T6 epidural sensory block. They found that EDA did not significantly alter renal blood flow [74]. In fact, 
EDA using lidocaine or bupivacaine is the preferred anesthesia method in renal transplantations because it displays lower toxicity for the recipient and renal allograft [75].

Furthermore, a review of trials with randomization of intraoperative neuraxial blockade showed that EDA reduces postoperative mortality and other complications, such as renal failure [76]. This finding is further reinforced by a meta-analysis, which found that EDA, in addition to general anesthesia, reduced the incidence of perioperative acute renal failure in cardiac surgery [77]. Lidocaine may provide protection against IR injury by preventing miRNA dysregulation. A recent study found that lung IR injury caused miRNA dysregulation, while lidocaine reduced these changes [78]. Further studies should be conducted to examine how lidocaine regulates miRNA expression.

In contrast, a recent study found that EDA may be a risk factor for postoperative AKI after major hepatectomy [79]. While attempting to elucidate the relationship between anesthesia and clinical AKI, it was found that for major hepatectomies, AKI incidence was significantly higher among patients who received EDA as compared to those who did not, suggesting that EDA may contribute to negative postoperative clinical AKI outcomes for patients [79]. However, since no significant difference in AKI incidence was revealed in patients undergoing minor hepatectomies, contradictory data suggest that EDA may have a beneficial impact during some types of surgery, but not others. Further studies are required to elucidate the clinical outcomes of EDA on renal function in different types of surgery.

\section{Conclusion}

Certain anesthetics seem to exhibit protective effects in patients through anti- inflammatory, anti-necrotic, and anti-apoptotic mechanisms. Additionally, intravenous anesthetics, such as propofol, are promising candidates for preventing or treating AKI, as well as IR injury. The effects of anesthetic drugs on renal function are particularly important in the context of kidney transplantation and preservation strategies. Although anesthetics may have important clinical implications, our understanding of the underlying mechanism of their renal protection is not fully understood. Further research is necessary to elucidate the molecular mechanisms of anesthetics, specifically those that contain lipids in their structure, to enable repurposing them for novel applications, such as transplant and organ-protective targets.

\section{Financial Disclosures}

The authors have nothing to disclose.

\section{Disclosure Statement}

The authors have no conflicts of interest to declare.

\section{Data Source}

Research in both human and animals has contributed the data needed for this study.

\section{References}

1 Gullick HD, Raizs LG: Changes in renal concentrating ability associated with major surgical procedures. N Engl J Med 1960;262:13091314.

2 Burchardi H, Kaczmarczyk G: The effect of anaesthesia on renal function. Eur J Anaesthesiol 1994;11:163-168.

3 Fukazawa K, Lee HT: Volatile anesthetics and AKI: risks, mechanisms, and a potential therapeutic window. J Am Soc Nephrol 2014;25: 884-892.

4 Sellbrant I, Brattwall M, Jildenstål P, Warren-Stomberg M, Forsberg S, Jakobsson JG: Anaesthetics and analgesics; neurocognitive effects, organ protection and cancer reoccurrence an update. Int J Surg 2016;34:4146.

5 Andrews DT, Royse C, Royse AG: The mitochondrial permeability transition pore and its role in anaesthesia-triggered cellular protection during ischaemia-reperfusion injury. Anaesth Intensive Care 2012;40:46-70.

6 L, Ruffenach G, Kararigas G, et al: Intralipid protects the heart in late pregnancy against ischemia/reperfusion injury via Caveolin2/ STAT3/GSK-3 $\beta$ pathway. J Mol Cell Cardiol 2017;102:108-116.

7 Li J, Motayagheni N, Barakati N, Eghbali M: Intralipid protects the heart in late pregnancy against ischemia/reperfusion injury by reducing cardiomyocyte apoptosis via Mir122 induction. Circ Res 2017;119:A442.

8 Motayagheni N, Eghbali M: Complete reversal of xylazine-induced bradycardia with intralipid in female mice. Circ Res 2016; 119:A253

9 Motayagheni N, Phan S, Eshraghi C, Eghbali M: Inhibition of leptin receptor abolishes in- tralipid-induced cardioprotection against ischemia-reperfusion injury. Cardiology 2016;134:241.

10 Motayagheni N, Eghbali M: Reversal of xylazine-induced bradycardia with intralipid. Cardiology 2016;134:431.

11 Motayagheni N: From bupivacaine to intralipid: Leading edge. J Anesth Clin Res 2016;4: 00164.

12 Li J, Motayagheni N, Barakati N, Eghbali M: Intralipid protects the heart against ischemia/ reperfusion injury by reducing cardiomyocyte apoptosis via miR122 induction in late pregnancy. Cardiology 2016;134:313.

13 Motayagheni N, Sharma S, Li J, Eghbali M: Implication of miR-1 and miR- 144 in intralipid-induced cardioprotection against ischemia/reperfusion injury. Cardiology 2016; 134:430. 
14 Cousins MJ, Mazze RI: Methoxyflurane nephrotoxicity. A study of dose response in man. JAMA 1973;225:1611-1616.

15 Conzen PF, Nuscheler M, Melotte A, et al: Renal function and serum fluoride concentrations in patients with stable renal insufficiency after anesthesia with sevoflurane or enflurane. Anesth Analg 1995;81:569-575.

16 Cousins MJ, Greenstein LR, Hitt BA, Mazze RI: Metabolism and renal effects of enflurane in man. Anesthesiology 1976;44:44-53.

17 Hashiguchi $\mathrm{H}$, Morooka $\mathrm{H}$, Miyoshi $\mathrm{H}$, et al: Isoflurane protects renal function against ischemia and reperfusion through inhibition of protein kinases, JNK and ERK. Anesth Analg 2005;101:1584-1589.

18 Vianna PT, Castiglia YM, Braz JR, et al: Remifentanil, isoflurane, and preconditioning attenuate renal ischemia/reperfusion injury in rats. Transplant Proc 2009;41:4080-4082.

19 Bracco D: Post-conditioning: promising answers and more questions. Crit Care 2012;16: 180.

20 Steurer MP, Steurer MA, Baulig W, et al: Late pharmacologic conditioning with volatile anesthetics after cardiac surgery. Crit Care 2012; 16:R191.

21 Murry CE, Jennings RB, Reimer KA: Preconditioning with ischemia: a delay of lethal cell injury in ischemic myocardium. Circulation 1986;74:1124-1136.

22 Gidday JM: Cerebral preconditioning and ischaemic tolerance. Nat Rev Neurosci 2006; 7:437-448.

23 Zhao ZQ, Corvera JS, Halkos ME, et al: Inhibition of myocardial injury by ischemic postconditioning during reperfusion: comparison with ischemic preconditioning. Am J Physiol Heart Circ Physiol 2003;285:H579-H588.

24 Feng J, Fischer G, Lucchinetti E, et al: Infarctremodeled myocardium is receptive to protection by isoflurane postconditioning: role of protein kinase B/Akt signaling. Anesthesiology 2006;104:1004-1014.

25 Ohsumi A, Marseu K, Slinger P, et al: Sevoflurane attenuates ischemia-reperfusion injury in a rat lung transplantation model. Ann Thorac Surg 2017;103:1578-1586.

26 Fleisher LA, Beckman JA, Brown KA, et al: ACC/AHA 2007 Guidelines on perioperative cardiovascular evaluation and care for noncardiac surgery: executive summary: a report of the American College of Cardiology/American Heart Association Task Force on Practice Guidelines (writing committee to revise the 2002 guidelines on perioperative cardiovascular evaluation for noncardiac surgery) developed in collaboration with the American Society of Echocardiography, American Society of Nuclear Cardiology, Heart Rhythm Society, Society of Cardiovascular Anesthesiologists, Society for Cardiovascular Angiography and Interventions, Society for Vascular Medicine and Biology, and Society for Vascular Surgery. J Am Coll Cardiol 2007;50:17071732 .
27 Gentz BA, Malan TP: Renal toxicity with sevoflurane: a storm in a teacup? Drugs 2001; 61;2155-2162.

28 Baxi V, Jain A, Dasgupta D: Anaesthesia for renal transplantation: an update. Indian J Anaesth 2009;53:139-147.

29 Kobayashi Y, Ochiai R, Takeda J, Sekiguchi H, Fukushima K: Serum and urinary inorganic fluoride concentrations after prolonged inhalation of sevoflurane in humans. Anesth Analg 1992;74:753-757.

30 Faure A, Bruzzese L, Steinberg JG, et al: Effectiveness of pure argon for renal transplant preservation in a preclinical pig model of heterotopic autotransplantation. J Transl Med 2016;14:40.

31 Cai J, Xu R, Yu X, Fang Y, Ding X: Volatile anesthetics in preventing acute kidney injury after cardiac surgery: a systematic review and meta-analysis. J Thorac Cardiovasc Surg 2014;148:3127-3136.

32 Liang Y, Li Z, Mo N, et al: Isoflurane preconditioning ameliorates renal ischemia-reperfusion injury through antiinflammatory and antiapoptotic actions in rats. Biol Pharm Bull 2014;37:1599-1605.

33 Kim M, Ham A, Kim JY, et al: The volatile anesthetic isoflurane induces ecto-5'-nucleotidase (CD73) to protect against renal ischemia and reperfusion injury. Kidney Int 2013; 84:90-103.

34 Zheng B, Zhan Q, Chen J, Xu H, He Z: Sevoflurane pretreatment enhance HIF-2 $\alpha$ expression in mice after renal ischemia/reperfusion injury. Int J Clin Exp Pathol 2015;8:1311413119.

35 Jia P, Teng J, Zou J, et al: Xenon protects against septic acute kidney injury via miR-21 target signaling pathway. Crit Care Med 2015; 43:e250-e259.

36 Dal Molin SZ, Kruel CR, De fraga RS, Alboim C, De oliveira JR, Alvares-da-silva MR: Differential protective effects of anaesthesia with sevoflurane or isoflurane: an animal experimental model simulating liver transplantation. Eur J Anaesthesiol 2014;31:695-700.

37 Beck-Schimmer B, Breitenstein S, Bonvini JM, et al: Protection of pharmacological postconditioning in liver surgery: results of a prospective randomized controlled trial. Ann Surg 2012;256:837-844; discussion 844-845.

38 Julier K, da Silva R, Garcia C, et al: Preconditioning by sevoflurane decreases biochemical markers for myocardial and renal dysfunction in coronary artery bypass graft surgery: a double-blinded, placebo-controlled, multicenter study. Anesthesiology 2003;98:1315-1327.

39 Lee JH, Joo DJ, Kim JM, Park JH, Kim YS, Koo $\mathrm{BN}$ : Preconditioning effects of the anesthetic administered to the donor on grafted kidney function in living donor kidney transplantation recipients. Minerva Anestesiol 2013;79: 504-514.

40 Louvier N, Lançon JP: [Do halogenated anesthetics protect from ischemic and reperfusion myocardial injuries?]. Ann Fr Anesth Reanim 1994;13:690-698.
41 Lee HT, Ota-Setlik A, Fu Y, Nasr SH, Emala CW: Differential protective effects of volatile anesthetics against renal ischemia-reperfusion injury in vivo. Anesthesiology 2004;101: 1313-1324.

42 Daqing M, Lim T, Xu J, et al: Xenon preconditioning protects against renal ischemic-reperfusion injury via hif-1alpha activation. Am Soc Nephrol 2009;20:713-720.

43 Guye ML, Mc Gregor B, Weil G, Arnal F, Piriou V: [Ischaemic and pharmacologic preconditioning: desflurane reduces renal reperfusion injury in rabbits]. Ann Fr Anesth Reanim. 2010;29:518-523.

44 Basile DP, Anderson MD, Sutton TA: Pathophysiology of acute kidney injury. Compr Physiol 2012;2:1303-1353.

45 Chang EI, Zarate MA, Rabaglino MB, et al: Ketamine suppresses hypoxia-induced inflammatory responses in the late-gestation ovine fetal kidney cortex. J Physiol 2016;594: 1295-1310

46 Yuzer H, Yuzbasioglu MF, Ciralik H, et al: Effects of intravenous anesthetics on renal ischemia/reperfusion injury. Ren Fail 2009;31: 290-296.

47 Dogan Z, Yuzbasioglu MF, Kurutas EB, et al: Thiopental improves renal ischemia-reperfusion injury. Ren Fail 2010;32:391-395.

48 Li Y, Liu S: The effect of dexmedetomidine on oxidative stress response following cerebral ischemia-reperfusion in rats and the expression of intracellular adhesion molecule-1 (ICAM-1) and S100B. Med Sci Monit 2017; 23:867-873.

49 Vasileiou I, Xanthos T, Koudouna E, et al: Propofol: a review of its non-anaesthetic effects. Eur J Pharmacol 2009;605:1-8.

50 Eroglu A: The effect of intravenous anesthetics on ischemia-reperfusion injury. Biomed Res Int 2014;2014:821513.

51 Erturk E: Ischemia-reperfusion injury and volatile anesthetics. Biomed Res Int 2014; 2014:526301.

52 Kato R, Foex P: Myocardial protection by anesthetic agents against ischemia-reperfusion injury: an update for anesthesiologists. Can J Anaesth 2002;49:777-791.

53 Yuzbasioglu MF, Aykas A, Kurutas EB, Sahinkanat T: Protective effects of propofol against ischemia/reperfusion injury in rat kidneys. Ren Fail 2010;32:578-583.

54 Li Y, Zhong D, Lei L, et al: Propofol prevents renal ischemia-reperfusion injury via inhibiting the oxidative stress pathways. Cell Physiol Biochem 2015;37:14-26.

55 Basu S, Meisert I, Eggensperger E, Krieger E, Krenn CG: Time course and attenuation of ischaemia-reperfusion induced oxidative injury by propofol in human renal transplantation. Redox Rep 12007;2:195-202.

56 Sanchez-Conde P, Rodriguez-Lopez JM, Nicolas JL, et al: The comparative abilities of propofol and sevoflurane to modulate inflammation and oxidative stress in the kidney after aortic cross-clamping. Anesth Analg 2008; 106:371-378 
57 Wang HH, Zhou HY, Chen CC, Zhang XL, Cheng G: Propofol attenuation of renal ischemia/reperfusion injury involves heme oxygenase-1. Acta Pharmacol Sin 2007;28:11751180.

58 Yoo YC, Yoo KJ, Lim BJ, et al: Propofol at tenuates renal ischemia-reperfusion injury aggravated by hyperglycemia. J Surg Res 2013; 183:783-791.

59 Assad AR, Delou JM, Fonseca LM, et al: The role of KATP channels on propofol preconditioning in a cellular model of renal ischemiareperfusion. Anesth Analg 2009;109:14861492.

60 Yang S, Chou WP, Pei L: Effects of propofol on renal ischemia/reperfusion injury in rats. Exp Ther Med 2013;6:1177-1183.

61 Yoo YC, Shim JK, Song Y, Yang SY, Kwak YL: Anesthetics influence the incidence of acute kidney injury following valvular heart surgery. Kidney Int 2014;86:414-422.

62 Leite TT, Macedo E, Martins Ida S, Neves FM, Liborio AB: Renal outcomes in critically ill patients receiving propofol or midazolam. Clin J Am Soc Nephrol 2015;10:19371945.

63 Luo C, Yuan D, Li X, et al: Propofol attenuated acute kidney injury after orthotopic liver transplantation via inhibiting gap junction composed of connexin 32. Anesthesiology 2015;122:72-86.

64 Ge M, Luo G, Yao W, et al: Propofol pretreatment attenuates remote kidney injury induced by orthotopic liver autotransplantation, which is correlated with the activation of
Nrf2 in rats. Mol Med Rep 2015;11:39623968.

65 Hao W, Zhao ZH, Meng QT, et al: Propofol protects against hepatic ischemia/reperfusion injury via miR-133a-5p regulating the expression of MAPK6. Cell Biol Int 2017; 41:495-504.

66 Snoeijs MG, Vaahtera L, de Vries EE, et al: Addition of a water-soluble propofol formulation to preservation solution in experimental kidney transplantation. Transplantation 2011;92:296-302.

67 Ko JS, Gwak MS, Choi SJ, et al: The effects of desflurane and propofol-remifentanil on postoperative hepatic and renal functions after right hepatectomy in liver donors. Liver Transpl 2008;14:1150-1158.

68 Kotake Y: Anesthetic protection against hepatic injury. Masui 2006;55:570-578.

69 Hatipoglu S, Yildiz H, Bulbuloglu E, et al: Protective effects of intravenous anesthetics on kidney tissue in obstructive jaundice. World J Gastroenterol 2014;20:3320-3326.

70 Pierce B, Bole I, Patel V, Brown DL: Clinical outcomes of remote ischemic preconditioning prior to cardiac surgery: a meta-analysis of randomized controlled trials. J Am Heart Assoc 2017;6:e004666.

71 Bang JY, Lee J, Oh J, Song JG, Hwang GS: The Influence of propofol and sevoflurane on acute kidney injury after colorectal surgery: a retrospective cohort study. Anesth Analg 2016;123:363-370.

72 Xue F, Zhang W, Chu HC: Assessing perioperative dexmedetomidine reduces the incidence and severity of acute kidney injury fol- lowing valvular heart surgery. Kidney Int 2016;89:1164.

73 Ross JD, Ripper R, Law WR, et al: Adding bupivacaine to high-potassium cardioplegia improves function and reduces cellular damage of rat isolated hearts after prolonged, cold storage. Anesthesiology 2006;105:746-752.

74 Suleiman MY, Passannante AN, Onder RL Greene-Helms WF, Perretta SG: Alteration of renal blood flow during epidural anesthesia in normal subjects. Anesth Analg 1997;84:10761080.

75 Solonynko I, Loba M, Orel J, Kobza I, Zhuk R, Yeliseev G: Renal transplantation - choice of anesthesia. Wiad Lek 1997;50(suppl 1 pt 1):447-448

76 Rodgers A, Walker N, Schug S, et al: Reduction of postoperative mortality and morbidity with epidural or spinal anaesthesia: results from overview of randomised trials. BM] 2000;321:1493.

77 Bignami E, Landoni G, Biondi-Zoccai GG, et al: Epidural analgesia improves outcome in cardiac surgery: a meta-analysis of randomized controlled trials. J Cardiothorac Vasc Anesth 2010;24:586-597.

78 Rancan L, Simon C, Marchal-Duval E, et al: Lidocaine administration controls microRNAs alterations observed after lung ischemiareperfusion injury. Anesth Analg 2016;123: 1437-1447.

79 Kambakamba P, Slankamenac K, Tschuor C, et al: Epidural analgesia and perioperative kidney function after major liver resection. $\mathrm{Br}$ J Surg 2015;102:805-812. 